\title{
Slack Selection for Unintentional Islanding: Practical Validation in a Benchmark Microgrid
}

\author{
L. Reyes-Chamorro*, W. Saab ${ }^{\dagger}$, R. Rudnik ${ }^{\dagger}$, A. M. Kettner*, M. Paolone* and J.-Y. Le Boudec ${ }^{\dagger}$ \\ * Distributed Electrical Systems Laboratory, \{lorenzo.reyes, andreas.kettner, mario.paolone\}@epfl.ch \\ $\dagger$ Laboratory for Communications and Applications 2, \{wajeb.saab, roman.rudnik, jean-yves.leboudec $\} @$ epfl.ch \\ École Polytechnique Fédérale de Lausanne (EPFL), Lausanne, Switzerland
}

\begin{abstract}
Upon an intentional or emergency disconnection from the main grid, a microgrid is expected to continue working in islanded mode. Thus, (at least) one resource needs to act as slack and compensate for power variations to keep the power balance, and ensure the security of supply. Although several resources might be eligible to become slack, some are more suitable than others (energy storage systems in particular) depending on the state of both the resources and the grid before the islanding transition. In this paper, we validate a recently proposed method to select in real-time the best slack-candidate using an abstract representation of the internal state of the available resources. The same method can be used to actively switch the slack during islanded operation to accommodate the intrinsic stochastic nature of the microgrid's resources. Our main contribution is the validation of the method in a realscale microgrid, including a discussion of implementation and deployment aspects. To support our findings, we present extensive experimental results in different operating conditions.
\end{abstract}

Index Terms-Slack Bus, Islanding, Microgrids, Validation.

\section{INTRODUCTION}

A medium/low voltage microgrid hosting distributed generation and storage devices might be able to run autonomously, i.e. to work in islanded mode, depending on its available power and energy flexibility [1]. This situation occurs, for example, in case of natural disasters when the main grid suffers a farreaching failure (triggering the local micirogrid unintentional islanding), or for main grid maintenance/reinforcement (triggering the local microgrid intentional/programmed islanding) [2]. We assume a microgrid controlled by a microgrid operator, which can participate to the energy market as a distribution system operator (DSO) with the additional feature of being able to disconnect the microgrid from the main grid and operate it autonomously when needed. The microgrid operator should keep a reserve of power and energy that is large enough to: (i) compensate for the instantaneous power step, and (ii) keep the system operational after the disconnection.

To ensure the power reserve, one solution is to install a predefined resource that is sized to withstand large power variations occurring during disconnection. This solution typically considers that the predefined resource is installed close to the $\mathrm{PCC}^{1}$ [3]. In this connection, storage systems are an obvious choice, since they can operate in all four quadrants of the

\footnotetext{
This work is supported by the SNSF-NRP70 "Energy Turnaround" project.

${ }^{1}$ Here, the PCC (point of common coupling) is defined as the connection point between the microgrid and the main grid.
}

$P Q$ plane, thus offering more flexibility than, for instance, conventional generation (e.g., diesel generator sets). However, such a solution may force the operator to have an additional device tailored for this purpose and, in general, does not use the overall available flexibility of the system. Another solution is to take advantage of existing storage devices in the grid and use conventional power sharing strategies, such as droop control, to share the power step among them [4], [5]. For this to work properly, there is the need to adapt the power setpoints after the microgrid isolation, to avoid the DC-side limits to be violated [6]. For instance, if a storage is almost fully charged, the DC voltage will be close to its maximum allowed value and it will not be able to continue charging at high power. However, this internal control loop does not account for the state of the grid and might cause voltage or current violations at buses where storage resources are not connected.

Considering that - from the system's point of view the storage DC-side limits represent time-dependant activepower limits, an alternative solution can be considered [7]. To tackle this issue, a method for ranking resources in real-time according to their suitability for becoming slack was proposed in [8]. This method uses abstract information of the internal state of the resources as proposed in [9], [10] - information that is continuously updated several times per second.

In this paper, we validate this method in a practical implementation case. Specifically, we show how to adapt it to be deployed in a real-scale microgrid making special emphasis on how to orchestrate all the events taking place in the islanding maneuver. We present results of this implementation for different operating conditions and the impact of the decision process on the state of the grid.

The remainder of this paper is structured as follows. Section II introduces the problem to solve. In Section III, we present the proposed method and the respective assumptions. Section IV shows the experimental validation setup used for the experiments. In Section $\mathrm{V}$, the results of performing the islanding maneuver using our method are presented and discussed. Finally, the conclusions are drawn in Section VI.

\section{Problem Statement}

We consider a generic microgrid comprising heterogeneous resources such as distributed generation, distributed storage, and loads with and without the ability to perform demand response. We assume that, when the microgrid is connected to 


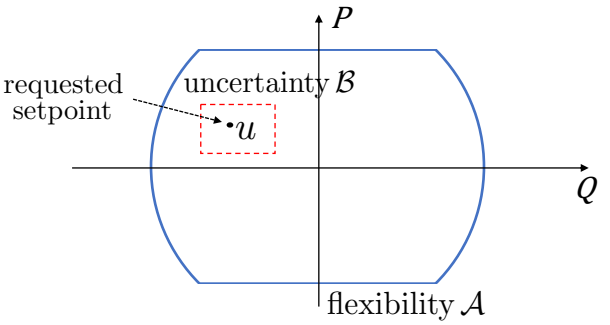

Figure 1. Representation of $\mathcal{A}$ and $\mathcal{B}$ for a battery resource.

the main grid, all resources work in grid-feeding mode, i.e. the injected/absorbed powers follow the amplitude and frequency of the voltage imposed by the main grid [11]. We assume that these devices are controlled by explicit power setpoints given by a system-level controller (i.e. not using droop control).

In this context, when the microgrid gets islanded, at least one resource must operate in grid-forming mode to impose constant voltage amplitude and frequency, i.e. it becomes the so-called slack. The system-level controller can mobilize a number of devices to maintain the power balance, but it is the slack that compensates the instantaneous imbalances that occur between two actions of the system-level controller. Here, we assume that only one resource should be slack at a time, and we leave for future work the multiple-slacks case.

\section{A. Slack-Candidate Ranking}

Our main goal is to identify and rank the resources $i \in \mathcal{R}$ that can become slack in the order of their ability to handle the islanding maneuver, being $\mathcal{R}$ the set of all resources in the microgrid. In this context, we consider that, when a resource $j$ is tracking a power setpoint $u_{j}$, its implemented complex power $x_{j}$ lies in a set defined by the function $\mathcal{B}_{j}\left(u_{j}\right)$ as in [9]. This function maps a point $u_{j}$ in the flexibility region $\mathcal{A}_{j}$ of the resource, to a set of points that the resource might implement if instructed to implement $u_{j} . \mathcal{A}_{j}$ is a subset of the $P Q$ plane in which the resource $j$ can operate.

As an example, Fig. 1 shows $\mathcal{A}$ and $\mathcal{B}$ of a battery at some point in time. The capped circle includes the set of all possible operation points, and the rectangle captures the setpoint deployment uncertainty: the battery will operate in this region if instructed to implement the requested setpoint.

Subsequently, when a resource $i$ becomes slack, it should be able to handle the resulting power uncertainty at its node produced by all other resources, while imposing the voltage phasor $v_{i}$. We define the mapping $\psi$, that computes a set of power flow (PF) solutions as follows:

$$
\begin{gathered}
\psi:\left\{u_{j}\right\} \times v_{i} \mapsto\left\{\operatorname{PF}\left(\left\{x_{j}\right\}, v_{i}\right): x_{j} \in \mathcal{B}_{j}\left(u_{j}\right), j \in \mathcal{R}_{-i}\right\} \\
\mathrm{PF}:\left\{x_{j}\right\} \times v_{i} \mapsto \mathbf{v} \times x_{i}, j \in \mathcal{R}_{-i}
\end{gathered}
$$

where $\mathbf{v}$ is the vector of voltage phasors and $\mathcal{R}_{-i}:=\mathcal{R} \backslash\{i\}^{2}$. The mapping $\psi$ computes the set of all possible resulting grid states $\{\mathbf{v}\}$ and the resulting powers $\left\{x_{i}\right\}$, when $i$ is slack, as a function of the combined uncertainty of all resources.

\footnotetext{
${ }^{2}$ For this, we assume that the grid representation is in the Kron-reduced form [12] (i.e., every node of the reduced network contains a resource).
}

Note that, when $i$ becomes the slack, there might be the need to disconnect a subset of resources, $\mathcal{S}_{i} \subseteq \mathcal{R}_{-i}$, for keeping feasible operating conditions after the disconnection (i.e., load shedding). We denote by $\mathcal{T}_{i}$ the set of resources that remain connected, $\mathcal{T}_{i}=\mathcal{R}_{-i} \backslash \mathcal{S}_{i}$. This leads to the following definition.

Definition 1: A feasible partition $\left\{i, \mathcal{S}_{i}, \mathcal{T}_{i}\right\}$ of $\mathcal{R}$ is one that satisfies the following constraints:

$$
\begin{aligned}
\forall\left(\mathbf{v}, x_{i}\right) & \in \psi\left(\left\{\bar{u}_{j}\right\}, \hat{v}_{i}\right), \bar{u}_{j}= \begin{cases}u_{j} & j \in \mathcal{T}_{i} \\
(0,0) & j \in \mathcal{S}_{i}\end{cases} \\
\forall n & \in \mathcal{N},\left|V_{n}\right| \in\left[V^{\text {min }}, V^{\text {max }}\right] \\
\forall \ell & \in \mathcal{L},\left|I_{\ell}\right| \leq I_{\ell}^{\text {max }} \\
x_{i} & \in \mathcal{A}_{i},
\end{aligned}
$$

where $\mathcal{N}$ is the set of all nodes, $\mathcal{L}$ is the set of all lines, $\hat{v}_{i}$ is the present voltage at the node where resource $i$ is connected, $V^{\min }$ and $V^{\max }$ are the minimum and maximum allowed (and given) voltages magnitudes respectively, $I_{\ell}$ the resulting current in line $\ell$ that can be computed from the power flow solutions, and $I_{\ell}^{\max }$ is the ampacity of line $\ell$.

Definition 2: A resource $i$ is a slack candidate if there exists a feasible partition $\left\{i, \mathcal{S}_{i}, \mathcal{T}_{i}\right\}$.

When there are multiple slack candidates, the best should be selected. This selection varies according to the current operating condition of each resource, thus a rating is needed. Such rating will be the solution of the following problem:

$$
\underset{i}{\arg \min } \mu\left(\left\{u_{j}\right\},\left\{\mathcal{A}_{j}\right\},\left\{\mathcal{B}_{j}\right\}, \hat{v}, i\right)
$$

where $j \in \mathcal{R}$ and $\mu$ is a generic function that can differ depending on the grid operator goals. In practice, this function should aim to maximize the number of resources that stay connected in islanded mode, maximize the slack power margins, and reward a better grid quality of service.

\section{B. Islanding Maneuver}

We assume that, at the point of connection between the main grid and the microgrid, there is a circuit breaker controlled by a synchrocheck relay. In our case, this device is used to detect an imminent islanding condition. We assume that the synchrocheck informs the central controller before manoeuvring the breaker (thereby islanding the microgrid) and waits until an acknowledgement is received from the central controller. The timespan between these two events has to be kept strictly within a given time budget. Note that, in case the breaker opens without previous notice, a smooth transition cannot be guaranteed. Yet, the central controller can ask the best slackcandidate to become slack after noticing the disconnection by monitoring, for instance, the breaker status.

Once the best slack-candidate is chosen (Section II-A), our next goal is to perform the transition from grid-connected to islanded mode, when the synchrocheck informs the imminent disconnection. Our main criterion for such a transition is to have exactly one slack resource (in grid-connected mode, the main grid is the slack resource). But, in a distributed system, this cannot be guaranteed at all times [13]. Thus, we focus on maximizing the likelihood of succeeding in passing from grid-connected to islanded mode with one slack. 


\section{Method}

In order for the microgrid to switch from grid-connected to islanded mode and appropriately choose a slack resource, we keep a slack candidate list that is ordered with respect to a set of metrics. For handling the islanding maneuver, we define a second entity: the Slack Manager (SM), that will be in charge of solving the problems in Section II.

As mentioned earlier, the SM requires the following inputs: (i) the requested setpoint $u_{j}$, (ii) the uncertainty $\mathcal{B}_{j}\left(u_{j}\right)$ and flexibility $\mathcal{A}_{j}$ of each resource $j$, and (iii) the measured grid state $\hat{\mathbf{v}}$. In our case, we make use of the COMMELEC framework for controlling the grid [9]. In this framework, a Grid Agent (GA) computes setpoints several times per second, by using a set of advertisements received from the resources, together with the state of the grid received from a state estimation tool. An advertisement is composed by the previously defined quantities $(\mathcal{A}, \mathcal{B})$. Fig. 2 shows the interaction between the COMMELEC GA and the SM. We assume that both know the topology / electrical parameters of the grid, as well as the location of the controllable resources.

In this paper, we assume that the SM has the capability of remotely disconnecting a resource by opening the circuit breaker of the line at which the latter is connected.

\section{A. Assumptions}

For our validation, we assume that (i) sets $\mathcal{A}$ are convex and that (ii) the sets defined by $\mathcal{B}(u)$ are rectangular, as the computation of most metrics relies on this assumption in order to execute in real-time. The first assumption holds for all advertisements sent by the resources used in our validation. However, taking the largest inscribed convex polygon inside a non-convex set $\mathcal{A}$ is one conservative approach that the SM can take in case the assumption does not hold. The second assumption is ensured by taking the bounding box around the set defined by $\mathcal{B}(u)$. This approach is also conservative, as the uncertainty is being overestimated.

\section{B. Finding a Feasible Partition}

The problem of finding a feasible partition for a resource $i$ involves performing an infinite number of power flows for the entire uncertainty range of the other resources, as seen in Eq. (1). We use the method proposed in [14] to perform this task. Thus, considering the minimum values of $\left(P_{j}, Q_{j}\right)=u_{j}$, for all $j$, as an input to $\psi$, yields the lowest possible nodal voltages and line currents. Similarly, considered the maximum values of $u_{j}$ yields the highest possible voltages and currents.

Accordingly, we propose the following method to find a feasible partition for resource $i$ :

1) Start with $\mathcal{S}_{i}=\emptyset$

2) Solve PF (2) four times, using as input the minimum/maximum values of $P_{j}$ and $Q_{j}$, for all $j$

3) If (4), (5), (6), are satisfied, or if $\mathcal{S}_{i}=\mathcal{R}_{-i}$, stop

4) Otherwise, add a resource to $\mathcal{S}_{i}$ and repeat steps 2)-4)

From Definition 2, the slack candidates are the ones for which a feasible partition exists. Our method uses a heuristic approach to find one such partition, where in step 4), we add a

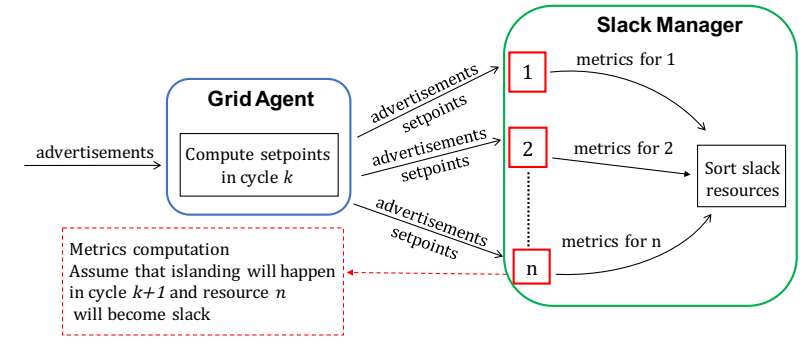

Figure 2. Computation of metrics for all resources and sorting the slack candidate list in each cycle.

resource to $\mathcal{S}_{i}$ based on some predefined priority. The resulting $\mathcal{S}_{i}$ is called the shedding list of resource $i$.

We define the three sets $\mathcal{B B}_{P}, \mathcal{B B}_{V}, \mathcal{B B}_{I}$ to include the results of the four power flows performed. $\mathcal{B B}_{P}$ includes the power at the slack bus, $\mathcal{B B}_{V}$ includes the maximum and minimum nodal voltages $\forall n \in \mathcal{N}$, and $\mathcal{B B}_{I}$ includes the maximum line currents $\forall \ell \in \mathcal{L}$. These sets will be used to compute some of the metrics described below.

\section{Metrics Computation}

The metrics discussed here are derived from [8]. They characterize the controllability, power availability, voltage and current feasibility, and survival time. The relevant assumptions considered to compute them and adopt them to a real infrastructure are discussed below.

The metrics for each resource are computed for a simulated scenario, in which islanding occurs before the next computation cycle, and the resource in question is chosen to be slack. The details of this process are shown in Fig. 2. The feasible partition of each slack candidate is used in the computation of its metrics. Based on those metrics, the ranking of the slack candidates is performed, which is taken into account when an actual islanding event occurs.

1) Controllability: Controllability is a binary metric used to additionally eliminate certain resource from consideration as a slack candidate (Definition 2). In [8], controllability $\rho_{C}(i)$ is defined as an indicator of whether the maximum uncertainty of the resource $i$ as described by its belief function, is less than some predefined threshold $\delta$ :

$$
\rho_{C}(i)= \begin{cases}1 & \max _{x \in \mathcal{A}_{i}} d\left(x, \mathcal{B}_{i}(x)\right)<\delta \\ 0 & \text { otherwise }\end{cases}
$$

The problem of computing controllability for generic belief functions appears to be undecidable, i.e. it belongs to a class of problems that are not guaranteed to halt [15], or at best intractable in real-time (recall that this method is to be performed several times per second).

Given that energy storage systems generally available in microgrids have a negligible uncertainty in deploying a given setpoint (depicted by an identity belief function that maps a point to itself), we reduced the controllability metric computation to a check as to whether or not the resource is an energy storage system. This is a characteristic that does not need to be continuously sent and it is in practice always known by a grid operator. It is also applicable to a diesel/gas turbine genset. 
2) Power Availability: The power availability metric $\rho_{P}(i)$ captures the safety margin of the ability of resource $i$ to handle the existing power imbalances if it becomes slack. It is computed, in relation to Eq. (6), as the minimum distance between the boundary of the flexibility $\left(\mathcal{A}_{i}\right)$ of the slack resource, and the set of all possible power injections at the slack resource. In practice, it suffices to check the four corners of the bounding box of the set of possible power flows:

$$
\rho_{P}(i)=\min _{x_{i} \in \mathcal{B B}_{P}} d\left(x_{i}, \mathcal{A}_{i}^{c}\right)
$$

where $\mathcal{A}_{i}^{c}$ is the complement of $\mathcal{A}_{i}$.

3) Feasibility: We consider two feasibility metrics: (i) The voltage feasibility metric $\rho_{V}(i)$ captures the safety margin of the nodal voltages with respect to some given bounds e.g. $\pm 10 \%$ of the nominal voltage magnitude. This is in relation to Eq. (4). (ii) The current feasibility metric $\rho_{I}(i)$ captures the safety margin of the line currents with respect to each line's ampacity, and is in relation to Eq. (5).

Using $V^{\max }, V^{\min }$ and $I_{\ell}^{\max }$ at each of the four corners of the bounding box, computed as shown earlier, we compute the feasibility metrics as follows:

$$
\begin{aligned}
\rho_{V}(i) & =\min _{V \in \mathcal{B B}_{V}} \min \left(\left|V^{\max }\right|-|V|,|V|-\left|V^{\text {min }}\right|\right) \\
\rho_{I}(i) & =\min _{I_{\ell} \in \mathcal{B B}_{I}}\left|I_{\ell}^{\text {max }}\right|-\left|I_{\ell}\right|
\end{aligned}
$$

4) Survival Time: Survival time $\rho_{s}(i)$ is a metric that measures the time a resource can withstand being slack, given its state of energy, capacity, and the current power flows in the grid. It is computed as the minimum of the charging and discharging survival times, as follows:

$$
\begin{aligned}
\rho_{s}(i) & =\min \left(\rho_{s c}(i), \rho_{s d}(i)\right) \\
\rho_{s c}(i) & =\min _{\left(P_{i}, Q_{i}\right) \in \mathcal{B} \mathcal{B}_{P}} \begin{cases}\left(1-\mathrm{SoE}_{i}\right) E_{i} / P_{i} & P_{i}<0 \\
\infty & \text { otherwise }\end{cases} \\
\rho_{s d}(i) & =\min _{\left(P_{i}, Q_{i}\right) \in \mathcal{B} \mathcal{B}_{P}} \begin{cases}\operatorname{SoE}_{i} E_{i} / P_{i} & P_{i}>0 \\
\infty & \text { otherwise }\end{cases}
\end{aligned}
$$

where $E_{i}$ is the rated energy of resource $i, \mathrm{SoE}_{i}$ is its fractional state-of-energy, and a positive $P_{i}$ represents discharging.

In addition to ranking the slack candidates, this metric is used to eliminate resources with $\rho_{s}(i)<\epsilon$, for some $\epsilon$, from being considered as slack candidates.

\section{Slack List}

After computing the metrics, the remaining slack candidates (after eliminating the non-storage devices and the resources with low survival-time) are sorted in decreasing order of $\left|\mathcal{T}_{i}\right|, \rho_{P}(i), \rho_{I}(i), \rho_{V}(i)$, and $\rho_{s}(i)$, lexicographically. The highest ranked resource is thus the best slack candidate. This ranking is one possible formulation of $\mu$ (Section II-B).

It is worth noting that the resource chosen to be slack is the one with the lowest number of devices to be shed. In case of a tie, the one with the highest safety margin of power availability is chosen. This is due to the fact that during the islanding maneuver, the slack will be required to absorb the power fluctuations and transients that occur. Voltage and current feasibility are given less priority since standards allow the violation of these bounds for short time intervals [16], [17].

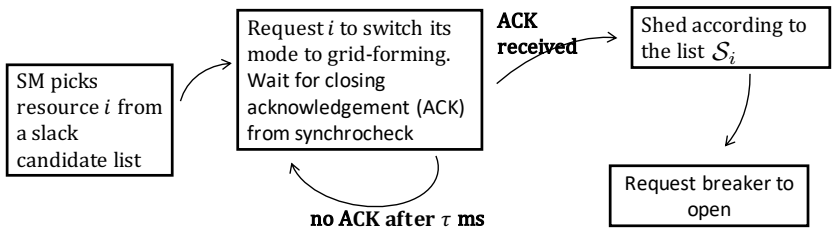

Figure 3. Islanding protocol: triggered upon detection of imminent islanding condition by synchrocheck.

\section{E. Islanding Protocol}

When the SM receives a notification of an imminent islanding condition from the synchrocheck, it follows the islanding protocol to perform a series of events required to transition from grid-connected to islanded mode. These events are: (1) requesting the best slack candidate $i$ to change its operation mode from grid-feeding to grid-forming, (2) disconnecting the elements from the shedding list $\mathcal{S}_{i}$, and (3) requesting the circuit breaker at the PCC to open.

We assume that, once the SM is notified of an imminent islanding condition, the circuit breaker at the PCC will be opened by the synchrocheck regardless of whether it receives a request from the SM or not. The SM can simply speed up the operation. In such a condition, the SM has to perform the steps of the islanding protocol in a time frame in the order of a few hundred milliseconds, we define this time as $T$ ms.

The protocol is described in detail in Fig. 3. The best slack candidate is requested to switch to grid-forming mode. Given that the communication between the SM and the resource might be affected by losses and delays, and due to the mission-critical nature of the islanding operation, this request is retransmitted every $\tau$ milliseconds ${ }^{3}$.

The maximum amount of retransmissions is, therefore, $n=\lceil T / \tau\rceil$. Given a packet loss probability of $p$ in the communication network, the probability of a successful islanding maneuver is thus $1-p^{n}$. When the operation is successful, and the new slack successfully acknowledges the mode switch, the resources in $\mathcal{S}_{i}$ are shed, and the breaker is requested to open.

The messages required for the islanding protocol are exchanged in between two COMMELEC cycles. In the presence of network delays (exacerbated by the use of retransmissions), the mode-switching messages from previous cycles might be received in subsequent cycles. Therefore, these messages are labelled with the sequence number of the COMMELEC cycle after which they occur. This enables the resources to discard messages that belong to older cycles.

\section{EXPERIMENTAL SETUP}

For the experimental validation, we use a real-scale (1:1) implementation of the benchmark low-voltage microgrid defined by the CIGRÉ Taskforce C6.04.02 [18], which is depicted in Fig. 4. The parts of the setup that are not used for the validation in this paper are greyed out. The selected setup complies with the minimum requirements for showing the features of the proposed method. In the following, the details

\footnotetext{
${ }^{3}$ In our setup, we take $\tau=2 \mathrm{~ms}$.
} 


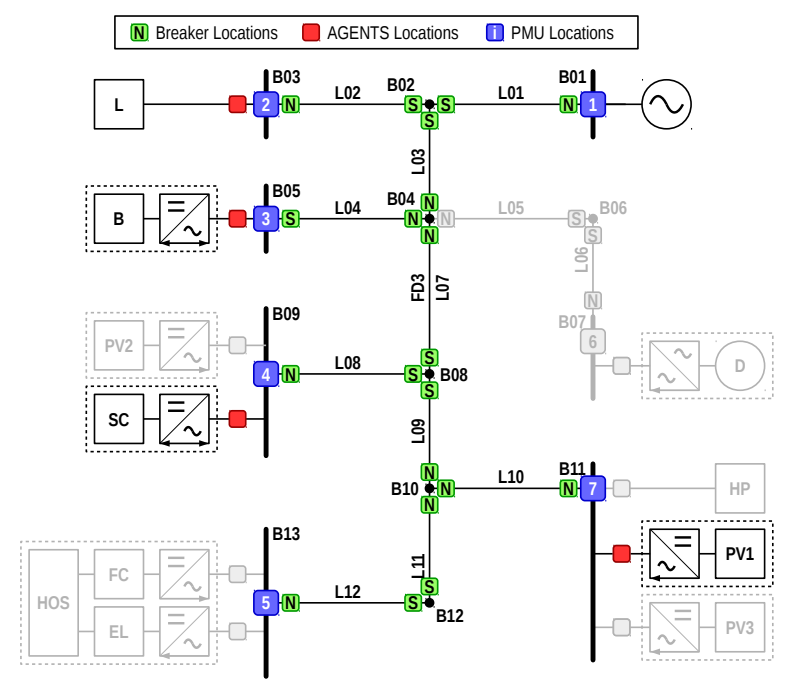

Figure 4. The experimental setup adapted from [18], [19]. In this paper, the following resources are used: the load (L), the battery (B), the supercapacitor bank (SC), and the photovoltaic panels (PV1). Those parts that are greyed out are not used for the validation.

of the implementation concerning the physical resources, the COMMELEC agents, and the islanding protocol are explained.

\section{A. Resources, Grid, and Instrumentation}

The following resources are part of the experimental setup used for the validation (see Fig. 4): (i) a $10 \mathrm{~kW} / 25 \mathrm{kWh}$ lithium-titanate battery cells (batteries), (ii) a $25 \mathrm{~kW} / 1 \mathrm{kWh}$ supercapacitor bank (Supercaps), (iii) a $7 \mathrm{~kW}$ photovoltaic plant (PV), and (iv) a $5.6 \mathrm{~kW}$ load, that simulates an electric heating system of 8 heaters of $700 \mathrm{~W}$ each. For more detailed information about the individual resources, please see [19].

Table I lists the cables' lengths and Cross-Sectional Areas (CSAs), and Table II their electrical parameters, i.e. perunit-length series resistance $R^{\prime}$ and reactance $X^{\prime}$, and rated current $I_{\max }$. For protection reasons, every cable is equipped with two circuit breakers, one at each end (labeled $\mathrm{N}$ for North / S for South). The breakers are configured to trip autonomously upon reaching the rated current of the line, but can also be ordered to do so using a control signal. In this setup, the breaker $\mathrm{N}$ of line L01 is operated in this manner to disconnect the microgrid from the local distribution grid. For operating the breaker, a dedicated software that follows the here-defined islanding protocol, has been deployed on a NI CompactRIO (model CRIO-9025).

The state of the grid is measured by Phasor Measurement Units (PMUs) also implemented on NI CRIOs (model CRIO-9063). Every PMU measures voltage and current in all three phases of its bus using class 0.1/0.2 sensors [20], [21], and streams synchrophasors at a refresh rate of 50 framesper-second. The phasor extraction algorithm is based on the interpolated discrete Fourier transform, and implemented into the field-programmable gate array (FPGA) of the CRIO [22]. To perform the time-alignment of the streamed synchrophasor measurements, a low-latency Phasor Data Concentrator (PDC) is employed [23]. The estimation of the grid state (i.e.,
TABLE I

Configuration of THE Benchmark Microgrid

\begin{tabular}{ccc} 
Name & Length $(\mathrm{m})$ & CSA $\left(\mathrm{mm}^{2}\right)$ \\
\hline L01 & 70 & 70 \\
L02 & 30 & 6 \\
L03 & 35 & 70 \\
L04 & 30 & 25 \\
L07 & 70 & 35 \\
L08 & 30 & 70 \\
L09 & 105 & 16 \\
L10 & 30 & 6 \\
L11 & 35 & 16 \\
L12 & 30 & 16
\end{tabular}

TABLE II

Electrical Parameters of the Low-Voltage Cables

\begin{tabular}{cccc}
$\mathrm{CSA}\left(\mathrm{mm}^{2}\right)$ & $R^{\prime}(\Omega / \mathrm{km})$ & $X^{\prime}(\Omega / \mathrm{km})$ & $I_{\max }(\mathrm{A})$ \\
\hline 6 & 3.300 & 0.141 & 44 \\
16 & 1.210 & 0.132 & 82 \\
25 & 0.780 & 0.126 & 108 \\
35 & 0.554 & 0.123 & 135 \\
70 & 0.272 & 0.119 & 207
\end{tabular}

the nodal voltage phasors) is performed using a linear State Estimator (SE) based on a Kalman Filter [24]. Both the PDC and the SE are implemented in NI LabVIEW, and executed on a desktop computer running Scientific Linux 7.

\section{B. Grid and Resource Agents}

Each of the resources previously described in Section IV-A is equipped with a Resource Agent (RA), which implements the functionality of the COMMELEC framework [9], [10]. The RAs are all implemented in NI LabVIEW and deployed into NI CRIOs (model CRIO-9068). For further information about the technical details of the RAs, please consult [19]. The Grid Agent (GA) and the slack manager are implemented in $\mathrm{C}++$, and executed on the same desktop machine where the PDC and the SE are located.

\section{RESULTS}

For validating the proposed method, we have conducted two experiments. On each experiment we intentionally modify the characteristics of the resources to show how the islanding maneuver is affected. A video of one experiment showing in real-time the metrics and the system state, when the islanding maneuver takes place, can be found on smartgrid.epfl.ch/?q=is landing.

\section{A. Best Slack Resource: Supercaps}

In the first experiment, the system objectives are the following: (i) the batteries to be discharged, (ii) the supercaps to be charged, (iii) the load needs little power to keep the emulated building temperature in the comfort zone, and (iv) the microgrid is set to export 5kW.As shown in Fig. 5, at the beginning of the experiment, the GA quickly finds the optimal operation point of the grid while fulfilling all the defined objectives. 

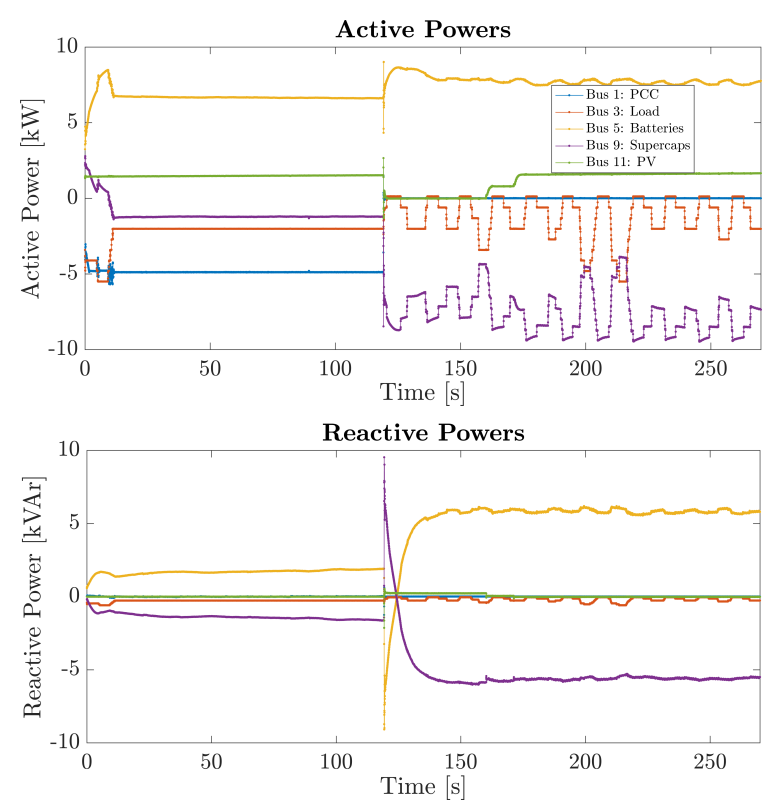

Figure 5. Islanding maneuver when the Supercaps resource becomes slack.
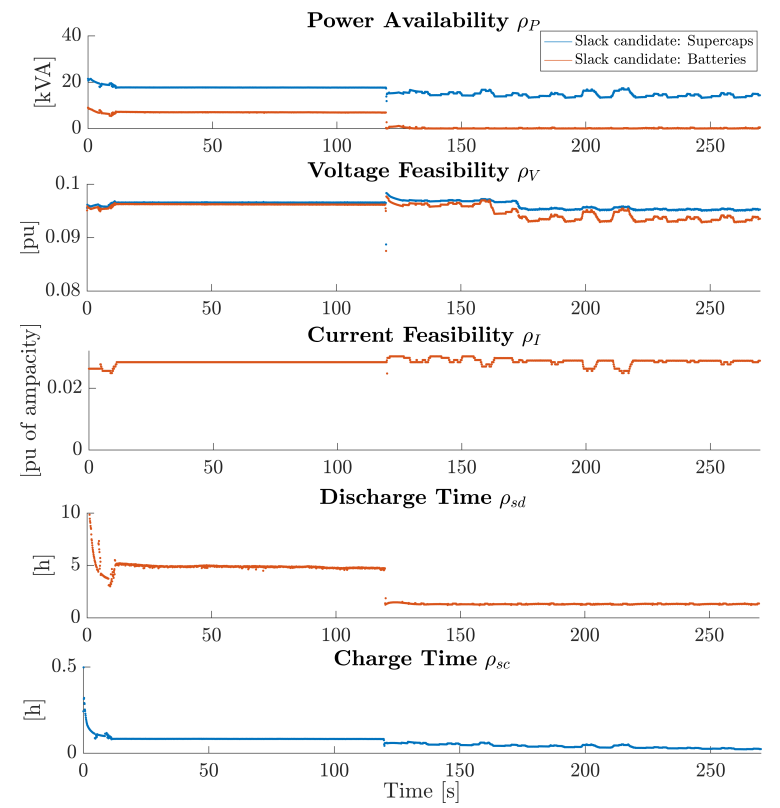

Figure 6. Islanding metrics when the Supercaps resource becomes slack.

We manually emulate the signal from the synchrocheck to the SM at around $t=120 \mathrm{~s}$, triggering the Islanding Protocol of Section III-E. In Fig. 6, the time-series of the islanding metrics (Section III-C) for this experiment are presented. Right before unexpected microgrid isolation, the best candidate slack is the supercaps resource (connected at bus 9).

A feasible partition was found, for both the batteries and the Supercaps, in which no resource is shed. Also, from Figure 6 , the Charge Survival Time of the supercaps is above the predefined threshold of $\epsilon=2 \mathrm{~min}$, and the Discharge Survival Time is infinite since the current implementation will not result in the supercaps discharging. The batteries have a high Survival Time, owing to the large capacity. Therefore, both the batteries and the supercaps are slack candidates.
The Power Availability metric thus becomes the deciding factor in the ranking, resulting in the supercaps being chosen. It can also be seen that the Voltage Feasibility metric is slightly better for the supercaps resource.

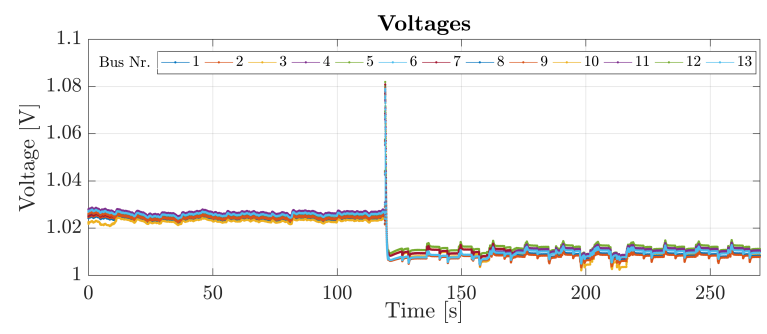

Figure 7. Voltage profiles when the Supercaps resource becomes slack.

After the completion of the Islanding Protocol, the supercaps resource became slack and the microgrid was fully disconnected from the main grid. This situation has caused the power jump of $5 \mathrm{~kW}$ in the microgrid that were being injected (before the isolation) into the main grid. As the new slack resource is not connected at the PCC, and it can control instantaneously only the voltage at its own node, the voltage profile of the microgrid is strongly affected, as seen in Fig. 7, requesting the immediate reaction of both batteries and supercaps to consume and inject reactive power, respectively, as seen in Fig. 5.

Note that, when the microgrid gets islanded, the PV production also drops. This is due to the fact that we are using commercial PV converters that are programmed to be disconnected when they detect drastic changes on the terminal voltage. The PV power is soon restored when they reconnect after around 40s. With the microgrid islanded, and the PV injection lost, the GA needs to find a new equilibrium and it starts making use of the flexibility of the load resource. The supercaps, as slack, will follow to keep the power balance on both active and reactive power.

Also note from Fig. 6, that the Charge Time metric for the supercaps is small, given their small energy reservoir. However, the GA will account for the willingness of the supercaps to have a state of charge close to $50 \%$, for computing setpoints to the batteries and the load. This maximizes the time that the supercaps can withstand being the slack.

\section{B. Best Slack Resource: Batteries}

In this experiment, we intentionally modify the rated power of the supercaps from $25 \mathrm{kVA}$ to $10 \mathrm{kVA}$ so that it is comparable with the rated power of the batteries. In practice, this is done by just changing a parameter in the resource agent (without any hardware or software intervention), hence advertising a smaller flexibility to the GA and SM. Here, we keep the same objectives as in the first case.

Fig. 8 shows the metrics for this case, clearly showing that the best slack candidate are the batteries, due to having a better Power Availability metric. In this scenario, even though both the batteries and the supercaps resources had the same rated power ( $25 \mathrm{kVA})$, the batteries would have had to deal with less imbalances than the supercaps in case an islanding condition occurred. For this reason, the batteries are the preferred slack 


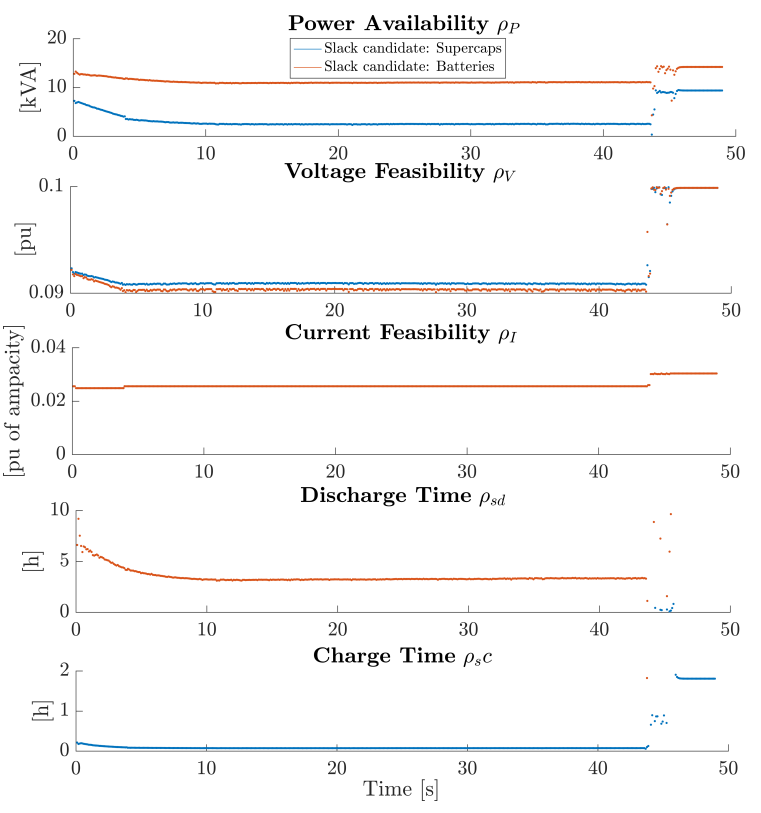

Figure 8. Islanding Metrics when the Batteries resource becomes slack.

candidate. The power profile for this experiment is similar to the previous one and can be hence omitted.

\section{CONCLUSION}

In this paper, we show the experimental validation of two methods capable to handle the microgrid islanding. The first one aims at selecting the resource that is better suited to become slack when a microgrid gets isolated. The second one looks for performing in practice the islanding maneuver by managing all the participant resources. We show through experiments performed in a real-scale implementation of the CIGRÉ C6.04.02 microgrid benchmark has been realized, that the Slack Manager continuously rates the different slack candidates according to the behaviour and characteristics of all the resources in the microgrid. The results for the first experiment show that, when the microgrid is exporting $5 \mathrm{~kW}$, it is possible to properly identify the best slack: in this case the supercapacitor bank. At the moment of the isolation from the main grid, the Slack Manager is able to keep the system operating in islanded mode, with neither voltage (within $\pm 10 \%$ ) nor current violations during the transition, and performs the proposed Islanding Protocol in less than $100 \mathrm{~ms}$. In this case, the supercapacitors are selected mainly because their rated power is larger than that of the battery. We also show that, when the supercapacitors rated power is equalized to that of the batteries, the batteries are ranked higher and thus selected to become slack, confirming the adaptability of the method and its capability to be applied in real-time. As future work, we plan to perform a re-synchronization maneuver. As in islanded mode the slack resource is connected to any node of the microgrid, we cannot control directly the voltage at the PCC to match the external one. Yet, as we perform a fixed frequency control, the frequency can be directly controlled at the slack resource, while voltage magnitude and phase are affected by the power flows. Thus, we can make use of the distributed resources to remain close to the main grid's voltage.

\section{REFERENCES}

[1] P. C. Loh, Y. K. Chai, D. Li, and F. Blaabjerg, "Autonomous Operation of Distributed Storages in Microgrids," IET Power Electronics, vol. 7, no. 1, pp. 23-30, January 2014.

[2] C. Chen, J. Wang, F. Qiu, and D. Zhao, "Resilient Distribution System by Microgrids Formation After Natural Disasters," IEEE Trans. Smart Grid, vol. 7, no. 2, pp. 958-966, March 2016.

[3] J. Rocabert, G. Azevedo, I. Candela, R. Teoderescu, P. Rodriguez, and I. Etxebarria-Otadui, "Microgrid Connection Management based on an Intelligent Connection Agent," in IECON 2010 - 36th Annual Conference of IEEE Ind. Electron. Society, Nov 2010, pp. 3028-3033.

[4] Q. Fu, A. Nasiri, V. Bhavaraju, A. Solanki, T. Abdallah, and D. C. Yu, "Transition Management of Microgrids With High Penetration of Renewable Energy," IEEE Trans. Smart Grid, vol. 5, no. 2, pp. 539549, March 2014

[5] J. M. Bloemink and M. R. Iravani, "Control of a Multiple Source Microgrid With Built-in Islanding Detection and Current Limiting," IEEE Trans. Power Del., vol. 27, no. 4, pp. 2122-2132, Oct 2012.

[6] N. Korada and M. K. Mishra, "Grid Adaptive Power Management Strategy for an Integrated Microgrid With Hybrid Energy Storage," IEEE Transactions on Industrial Electronics, vol. 64, no. 4, pp. 2884-2892, April 2017.

[7] M. Bahramipanah, D. Torregrossa, R. Cherkaoui, and M. Paolone, "An adaptive model-based real-time voltage control process for active distribution networks using battery energy storage systems," in 2016 Power Systems Computation Conference (PSCC), June 2016, pp. 1-8.

[8] A. Bernstein, L. Reyes-Chamorro, J.-Y. Le Boudec, and M. Paolone, "Real-Time Control of Microgrids with Explicit Power Setpoints: Unintentional Islanding," in PowerTech 2015, vol. 125, 2015, pp. 265-280.

[9] — - "A Composable Method for Real-Time Control of Active Distribution Networks with Explicit Power Setpoints. Part I: Framework," Elect. Power Syst. Research, vol. 125, pp. 254-264, 2015.

[10] L. Reyes-Chamorro, A. Bernstein, J.-Y. Le Boudec, and M. Paolone, "A Composable Method for Real-Time Control of Active Distribution Networks with Explicit Power Setpoints. Part II: Implementation and Validation," Elect. Power Syst. Research, vol. 125, pp. 265-280, 2015.

[11] J. Rocabert, A. Luna, F. Blaabjerg, and P. Rodriguez, "Control of Power Converters in AC Microgrids," IEEE Trans. Power Electron., vol. 27, no. 11, pp. 4734-4749, Nov. 2012

[12] G. Kron, Tensors for Circuits, 2nd ed. Mineola, NY, USA: Dover Publications, 1959.

[13] M. J. Fischer, N. A. Lynch, and M. S. Paterson, "Impossibility of Distributed Consensus with One Faulty Process," Journal of the ACM (JACM), vol. 32, no. 2, pp. 374-382, 1985.

[14] C. Wang, J.-Y. Le Boudec, and M. Paolone, "Controlling the Electrical State via Uncertain Power Injections in ThreePhase Distribution Networks," IEEE Trans. Smart Grid, 2017, DOI:10.1109/TSG.2017.2764077.

[15] M. Sipser, Introduction to the Theory of Computation. Thomson Course Technology Boston, 2006, vol. 2.

[16] IEEE Power and Energy Society, "IEEE Guide for Voltage Sag Indices," 2014.

[17] IEEE Power Engineering Society, "IEEE Standard for Calculating the Current-Temperature of Bare Overhead Conductors," 2006.

[18] Taskforce C6.04.02, "Benchmark Systems for Network Integration of Renewable and Distributed Energy Resources," CIGRÉ, Paris, IDF, FR, Tech. Rep. 575, 2010.

[19] L. Reyes-Chamorro, "Real-Time Control Framework for Active Distribution Networks - Theoretical Definition and Experimental Validation," Dissertation, École Polytechnique Fédérale de Lausanne, Faculté Sciences et Techniques de l'Ingénieur, Lausanne, VD, CH, 2016.

[20] "Instrument Transformers. Part 1: General Requirements," 2010, IEC Standard 61869-1.

[21] "Instrument Transformers. Part 2: Additional Requirements for Current Transformers," 2010, IEC Standard 61869-2.

[22] P. Romano and M. Paolone, "Enhanced Interpolated-DFT for Synchrophasor Estimation in FPGAs: Theory, Implementation, and Validation of a PMU Prototype," IEEE Trans. Instrum. Meas., vol. 63, no. 12, pp. 2824-2836, 2014.

[23] A. Derviškadić, P. Romano, M. Pignati, and M. Paolone, "Architecture and Experimental Validation of a Low-Latency Phasor Data Concentrator," IEEE Trans. Smart Grid, 2016, DOI: 10.1109/TSG.2016.2622725.

[24] A. M. Kettner and M. Paolone, "Sequential Discrete Kalman Filter for Real-Time State Estimation in Power Distribution Systems: Theory and Implementation," IEEE Trans. Instrum. Meas., vol. 66, no. 9, pp. 23582370, Sept 2017. 